$11-1995$

\title{
Connection Between Conserved Quantities and Degeneracies in Quantum Systems
}

\author{
S. Fallieros \\ Evangelos Hadjimichael \\ Fairfield University, ehadjimichael@fairfield.edu
}

Follow this and additional works at: https://digitalcommons.fairfield.edu/engineering-facultypubs

\section{Peer Reviewed}

\section{Repository Citation}

Fallieros, S. and Hadjimichael, Evangelos, "Connection Between Conserved Quantities and Degeneracies in Quantum Systems" (1995). Engineering Faculty Publications. 2.

https://digitalcommons.fairfield.edu/engineering-facultypubs/2

\section{Published Citation}

Fallieros, S. and Hadjimichael, E. 1995. Connection Between Conserved Quantities and Degeneracies in QuantumSystems. Am. J. Phys. 63, 11: 1017-1020.

This item has been accepted for inclusion in DigitalCommons@Fairfield by an authorized administrator of DigitalCommons@Fairfield. It is brought to you by DigitalCommons@Fairfield with permission from the rightsholder(s) and is protected by copyright and/or related rights. You are free to use this item in any way that is permitted by the copyright and related rights legislation that applies to your use. For other uses, you need to obtain permission from the rights-holder(s) directly, unless additional rights are indicated by a Creative Commons license in the record and/or on the work itself. For more information, please contact digitalcommons@fairfield.edu. 
reduction. Again, this is consistent with our measurements.

\section{EXPERIMENTAL RESULTS}

It is clear that in measuring the period of small oscillations of a set of partial rings, the significant sources of systematic error are (1) any radial offset of the pivot point from the center of the rings and (2) the effect of supporting the rings on a round peg rather than a knife edge. Both errors increase in significance as the half-angle of the rings is reduced. Our smallest half-angle was $\pi / 4$. Thus it is useful to examine the effects of these two sources of error for this smallest ring. Table I summarizes our results. The first column of the table lists the radial offset of the support point of the ring, the second column lists the radius of the support peg, and the third column lists the radius of the support hole. The fourth column gives the theoretical period for the ring including corrections for radial offset and support peg. Finally, the last column lists the measured period of the ring.

In terms of ring configuration, the first row in Table I represents a modified ring with the support hole filled in and a notch cut in the ring from below, halfway through the ring. The ring was then supported on a knife edge. Accordingly, it represents a ring with zero radial offset and a support peg with zero radius. The agreement between the theoretical and measured results is excellent. The second row in Table I shows the effect of moving the support point radially by 0.27 $\mathrm{cm}$. However, the support is still a knife edge. As can be seen, this radial offset introduces an approximate $4.4 \%$ reduction in the period. The third and fourth rows in Table I show the effect of introducing a round support peg of increasing radius. The larger peg of radius $0.122 \mathrm{~cm}$ in a hole of radius $0.25 \mathrm{~cm}$ introduces an additional reduction in period of about $3 \%$.
Finally, the last row in Table I shows the effect of filling in the support notch and mounting the ring on a knife edge along the inner radius of the ring. This amounts to a radial offset of $-0.50 \mathrm{~cm}$. In this case, the agreement between the theoretical and measurement periods was exact (no doubt by chance). In general, the agreement in the table between the theoretical values for the period and the measured values is quite good, although at the largest peg size there is a $2 \%$ discrepancy.

\section{CONCLUSIONS}

It is clear that a set of carefully constructed partial rings can make an excellent experiment for a physics laboratory. If the desire is to produce a set of rings that have observed periods independent of half-angle, then care must be taken to insure that (1) the rings all have the same radius, (2) the support point is accurately placed at the center of each ring (the point where the bisector and the mean radius of the ring intersect), and (3) the ring is supported on a knife edge rather than a round peg. To the extent that it is desired to introduce systematic errors into the experiment, one or more of these constraints may be intentionally relaxed.

\footnotetext{
${ }^{1}$ Cenco Operating Instructions (Central Scientific Company, Chicago, 1969), No. 071942-063 Cenco-Miller truncated-ring-pendulum paradox demonstrator.

${ }^{2}$ Thomas A. Walkiewicz and David L. Wagner, "Symmetry properties of a ring pendulum," Phys. Teach. 32, 142-144 (1994).

${ }^{3}$ Thomas A. Walkiewicz, "Experiment and theoretical analysis of a partial ring pendulum," AAPT Announcer 23, 90 (1993).

${ }^{4}$ David A. Giltinan (private communication).

${ }^{5}$ Alan Cromer, "Many oscillations of a rigid rod," Am. J. Phys. 63, 112 (1995).
}

\title{
Connection between conserved quantities and degeneracies in quantum systems
}

\author{
$\mathrm{S}$. Fallieros \\ Department of Physics, Brown University, Providence, Rhode Island 02912 \\ E. Hadjimichael \\ Department of Physics, Fairfield University, Fairfield, Connecticut 06430
}

(Received 19 July 1994; accepted 1 February 1995)

In the framework of quantum theory, we present one theorem and three corollaries regarding the direct connection between constants of motion of a physical system and degeneracies of its energy eigenvalues. It is shown that this connection emerges when there exist quantum operators which commute with the Hamiltonian, but not with each other. Further it is shown that if the commutator of these operators is a nonvanishing constant number then (a) all the eigenvalues of the system are degenerate, and (b) the degree of degeneracy is infinite. A number of examples are discussed including the parity degeneracy of the hydrogen atom and the infinite degeneracy of the Landau levels of a charged particle in a constant magnetic field. (C) 1995 American Association of Physics Teachers. 


\section{INTRODUCTION}

The hallmark of any physical theory dealing with the time development of a system is the identification of the system's constants of motion. In the quantum regime, these are conserved quantities, e.g., expectation values of Hermitian operators $\hat{O}$ which commute with the Hamiltonian of the system, $\hat{H}$. In other words, for the quantal constants of motion,

$$
d / d t\langle\hat{O}\rangle=i / \hbar\langle[\hat{H}, \hat{O}]\rangle=0 .
$$

It is also readily shown ${ }^{1}$ that the commutativity of $\hat{H}$ with an operator $\hat{O}$ leads to the invariance of $\hat{H}$ under a group of transformations generated by $\hat{O}$. This intimate relation between the invariances of the system under groups of transformations, and corresponding constants of the motion (conserved variables) is, of course, very well understood.

A related aspect of the vanishing of commutators, say, $\left[\hat{H}, \hat{O}_{1}\right]=0,\left[\hat{H}, \hat{O}_{2}\right]=0,\left[\hat{O}_{1}, \hat{O}_{2}\right]=0$, etc., is the existence of common eigenstates of $\hat{H}, \hat{O}_{1}, \hat{O}_{2}, \ldots$ This establishes the presence of a complete set of observables, corresponding to eigenvalues of the commuting operators, which specify the nature of the stationary states of the system. All these properties are thoroughly discussed in standard texts of quantum mechanics.

In this work, we discuss an additional relation involving constants of the motion on one hand, and the appearance of degeneracies in the eigenvalues of many observables, on the other. We trace these degeneracies to the commutator algebra of the operators corresponding to the constants of motion of the physical system.

In Sec. II of this paper, we discuss and prove general theorems which have a bearing on this problem. In Sec. III, we present applications to two systems of physical interest. We summarize our conclusions in Sec. IV.

\section{DEGENERACY OF EIGENSTATES AND CONSTANTS OF MOTION}

We discuss and prove here one theorem and three corollaries.

Theorem: Consider the Hamiltonian of a system, $\hat{H}$, having a set of eigenfunctions $\left|\Psi_{n}\right\rangle$ such that

$$
\hat{H}\left|\Psi_{n}\right\rangle=E_{n}\left|\Psi_{n}\right\rangle \text {. }
$$

Assume also the existence of a pair of Hermitian operators $\hat{O}_{1}$ and $\hat{O}_{2}$ corresponding to constants of the motion of the system, that is,

$$
\begin{gathered}
{\left[\hat{H}, \hat{O}_{1}\right]=0,} \\
{\left[\hat{H}, \hat{O}_{2}\right]=0,}
\end{gathered}
$$

which do not commute with each other

$$
\left[\hat{O}_{1}, \hat{O}_{2}\right]=\hat{C} \neq 0 \text {. }
$$

Equation (4) signifies an "incompatibility" between the two constants of motion corresponding to operators $\hat{O}_{1}$ and $\hat{O}_{2}$. Under those conditions, some of the eigenvalues of $\hat{H}$ are necessarily degenerate.

Proof: Suppose that one of the eigenstates of $\hat{H}$, with energy eigenvalue $E_{n}$, is not degenerate. Then, operating with $\hat{O}_{1}$ on Eq. (2), we obtain

$$
\hat{O}_{1} \hat{H}\left|\Psi_{n}\right\rangle=\hat{H} \hat{O}_{1}\left|\Psi_{n}\right\rangle=E_{n} \hat{O}_{1}\left|\Psi_{n}\right\rangle
$$

where the commutativity of $\hat{H}$ and $\hat{O}_{1}$ has been used. We see that both $\left|\Psi_{n}\right\rangle$ and $\hat{O}_{1}\left|\Psi_{n}\right\rangle$ are eigenstates of $\hat{H}$ with the same eigenvalue $E_{n}$; in order that eigenvalues $E_{n}$ be not degenerate, according to our assumption, the state $\hat{O}_{1}\left|\Psi_{n}\right\rangle$ must be proportional to $\left|\Psi_{n}\right\rangle$, i.e.,

$$
\hat{O}_{1}\left|\Psi_{n}\right\rangle=\alpha_{1}\left|\Psi_{n}\right\rangle,
$$

where $\alpha_{1}$ is a multiplicative constant. Similar arguments for the operator $\hat{O}_{2}$ lead to

$$
\hat{O}_{2}\left|\Psi_{n}\right\rangle=\alpha_{2}\left|\Psi_{n}\right\rangle \text {, }
$$

where $\alpha_{2}$ is another multiplicative constant. Hence, Eqs. (6) and (7) lead to

$$
\begin{aligned}
\hat{C}\left|\Psi_{n}\right\rangle & =\left(\alpha_{1} \alpha_{2}-\alpha_{2} \alpha_{1}\left|\Psi_{n}\right\rangle\right. \\
& =0 \text { for } E_{n} \text { nondegenerate. }
\end{aligned}
$$

If we now assume that all $\left|\Psi_{n}\right\rangle$ correspond to nondegenerate eigenvalues, then we may use the completeness of the spectrum to expand an arbitrary nonvanishing state $|\Psi\rangle$, viz.

$$
|\Psi\rangle=\sum_{n} c_{n}\left|\Psi_{n}\right\rangle
$$

Operating with the commutator $\hat{C}$, we find

$$
\hat{C}|\Psi\rangle=\sum_{n} c_{n} \hat{C}\left|\Psi_{n}\right\rangle=0,
$$

where we have used Eq. (8). The implication of Eq. (10) is that $\hat{C}=0$, in contradiction to the original assumption, Eq. (4). We conclude that, as a result of Eqs. (3) and (4), at least a part of the spectrum of $\hat{H}$ must be degenerate. This completes the proof of the theorem.

Corollary 1: If the commutator $\hat{C}$ in Eq. (4), is a constant number $c$, then the entire spectrum of $\hat{H}$ is degenerate. This is so, because Eq. (8) for eigenstates corresponding to nondegenerate eigenvalues cannot be satisfied for a nonvanishing constant number $c$.

Corollary 2: If, as above, the commutator $\hat{C}$ is a constant number $c$, the spectrum of eigenstates of $\hat{H}$ has an infinite degree of degeneracy. For, in evaluating the trace of the matrix elements of the product $\hat{O}_{1} \hat{O}_{2}$ in the subspace of degenerate states $\left|\Psi_{n, i}\right\rangle, i=1, \ldots, G$, with $G$ being the degree of degeneracy,

$$
\operatorname{Tr}\left(\hat{O}_{1} \hat{O}_{2}\right)=\sum_{i=1}^{G}\left\langle\Psi_{n, i}\left|\hat{O}_{1} \hat{O}_{2}\right| \Psi_{n, i}\right\rangle,
$$

the completeness of the set $\left|\Psi_{n, i}\right\rangle$ allows us to write

$$
\operatorname{Tr}\left(\hat{O}_{1} \hat{O}_{2}\right)=\sum_{i, j=1}^{G}\left\langle\Psi_{n, i}\left|\hat{O}_{1}\right| \Psi_{n, j}\right\rangle\left\langle\Psi_{n, j}\left|\hat{O}_{2}\right| \Psi_{n, i}\right\rangle
$$

which is the trace of a product of two matrices. For $G=$ finite, this trace is independent of the order of the matrices, which leads to

$$
\sum_{i=1}^{G}\left\langle\Psi_{n, i}|\hat{C}| \Psi_{n, i}\right\rangle=0=c \sum_{i=1}^{G}\left\langle\Psi_{n, i} \mid \Psi_{n, i}\right\rangle
$$

This can only be true if $\hat{C}=c=0$, in contradiction to the original assumption, Eq. (4). Hence we conclude that $G=\infty$, i.e., the degree of degeneracy is infinite.

Corollary 3: If $\hat{O}_{1}$ and $\hat{O}_{2}$ anticommute, then one or the other of the two operators annihilates each eigenstate corresponding to a nondegenerate eigenvalue. This follows from 
the fact that, in this case, $\hat{C}=2 \hat{O}_{1} \hat{O}_{2}$, in conjunction with Eq. (8) which is valid when $E_{n}$ is nondegenerate.

We illustrate these ideas by three simple examples here, and in the following section we discuss two less well-known, but quite important, applications. A very familiar example is the degeneracy of angular momentum states associated with the central force problem. Recall that for a spherically symmetric potential the Hamiltonian $\hat{H}$ commutes with the angular momentum operator $\hat{\mathbf{L}}$, signifying the conservation of this latter. Hence, $\left[\hat{H}, \hat{L}^{2}\right]=0,[\hat{H}, \hat{L} z]=0$, and $\left[\hat{H}, \hat{L}_{ \pm}\right]=0$, where $\hat{L}_{ \pm}=\hat{L}_{x} \pm i \hat{L}_{y}$ are the raising and lowering operators, respectively. On the other hand, $\left[\hat{L}_{z}, \hat{L}_{ \pm}\right]= \pm \hbar L_{ \pm}$. Hence the conditions in Eqs. (3) and (4) are satisfied for the operators $\hat{H}, \hat{L}_{ \pm}, \hat{L}_{z}$. The consequences are well known: both $|E ; l, m\rangle$ and $\hat{L}_{ \pm}|E ; l, m\rangle$ are eigenfunctions of $\hat{H}$ with a different eigenvalue $m$ of $\hat{L}_{z}$, but corresponding to the same eigenvalues of energy and $\hat{L}^{2}, E$, and $l$, respectively; this then is the origin of the well-known degeneracy of energy and angular momentum eigenvalues in this example.

Further, by Corollary $3, \hat{L}_{z}$ annihilates every nondegenerate eigenstate of $\hat{H}$. The only one of this type is, of course, the $s$ state.

Further simple examples are the isotropic, twodimensional harmonic oscillator and the free particle. For the former, the Hamiltonian is $\hat{H}=\hat{H}_{1}+\hat{H}_{2}$, with

$$
\hat{H}_{i}=\hat{p}_{i}^{2} / 2 m+m \omega^{2} \hat{x}_{i}^{2} / 2, \quad \hat{x}_{i=1,2}=\hat{x}, \hat{y} .
$$

The conditions of Eqs. (3) and (4) are satisfied by operators $\hat{H}, \hat{H}_{1}$ (or $\hat{H}_{2}$ ) and the $z$ component of the angular momentum $\hat{L}_{z}=\hat{x} \hat{p}_{y}-\hat{y} \hat{p}_{x}$; that is, $\hat{H}$ commutes with $\hat{H}_{1}$ (or $\hat{H}_{2}$ ) and $\hat{L}_{z}$, but $\left[H_{1}, L_{z}\right] \neq 0$, leading to well-known degeneracies.

In the case of a free particle in one dimension, Eqs. (3) and (4) are satisfied by the Hamiltonian $\hat{H}=\hat{p}^{2} / 2 m$, the momentum $\hat{p}=-i \hbar \partial / \partial x$ and parity $\hat{P}$, both constants of the motion. In this case, the well-known solutions of opposite parity, $\cos k x$ and $\sin k x$, correspond to the same energy, $\hbar^{2} k^{2} / 2 m$, which is thus degenerate.

\section{APPLICATIONS}

We continue with two important applications of the theorem and its corollaries discussed in the previous section.

\section{A. An electron in a hydrogenlike atom}

Consider the nonrelativistic Hamiltonian

$$
\begin{aligned}
\hat{H} & =\hat{p}^{2} / 2 m-\kappa / r \\
& =L^{2} / 2 m r^{2}-\left(\hbar^{2} / 2 m r^{2}\right) \partial / \partial r r^{2} \partial / \partial r-\kappa / r
\end{aligned}
$$

with $\kappa=Z e^{2}$, for an electron in the Coulomb potential in a hydrogenlike atom. In addition to the conservation of the orbital angular momentum $\hat{\mathbf{L}}$ and parity $\hat{P}$, the direction of the major axis of classical elliptical orbits is also constant in time. The quantity of interest in this case is the Runge-Lenz vector. $^{2}$ The classical form of this vector is defined by

$$
\epsilon=\mathbf{p} \times \mathbf{L} / \kappa m-\mathbf{r} / r
$$

and the quantum version is

$$
\hat{\boldsymbol{\epsilon}}=(\hat{\mathbf{L}} \times \hat{\mathbf{p}}-\hat{\mathbf{p}} \times \hat{\mathbf{L}}) / 2 \kappa m-\mathbf{r} / r .
$$

In the classical regime the following properties of the Runge-Lenz vector are noteworthy: as seen from Eq. (16) and the fact that $\mathbf{L}$ is constant, (a) $d \epsilon / d t=0$, i.e., $\epsilon$ is a constant of the motion.

(b) $\boldsymbol{\epsilon} \cdot \mathbf{L}=0$, and in view of $\mathbf{r} \cdot \mathbf{L}=0, \boldsymbol{\epsilon}$ and $\mathbf{r}$, and therefore the particle orbit, are confined to a plane perpendicular to $\mathrm{L}^{2}$

(c) In terms of the angle $\phi$ between the position vector $\mathbf{r}$ and the Runge-Lenz vector $\epsilon$, defined by $\mathbf{r} \cdot \boldsymbol{\epsilon}$ $=r \epsilon \cos \phi$, and by means of Eq. (16), we find

$$
r=\left(L^{2} / \kappa m\right) /(1+\epsilon \cos \phi) .
$$

This is the equation of a conic-section orbit, with $\epsilon$, the magnitude of the Runge-Lenz vector, being the eccentricity of the orbit. These features are also present in the case of planetary orbits. $^{3}$

Turning to the quantum regime, it is straightforward to show that $\hat{\epsilon}$ commutes with the Hamiltonian $\hat{H},[\hat{H}, \hat{\epsilon}]=0$, so that its expectation value is a constant of the motion. In addition, parity $P$ is conserved since obviously $[\hat{H}, \hat{P}]=0$. However,

$$
[\hat{P}, \hat{\epsilon}]=\hat{C}=-2 \pi \hat{\boldsymbol{\epsilon}} \neq 0 .
$$

where $\pi= \pm 1$ are the eigenvalues of the parity operator. Hence, the Hamiltonian $\hat{H}$, the parity $\hat{P}$, and the RungeLenz operator $\hat{\epsilon}$ satisfy the conditions in Sec. II, Eqs. (3) and (4). We expect therefore, that there will be degeneracies in the spectrum of energy eigenstates. Indeed, operating on

$$
\hat{H}|E, \pi\rangle=E|E, \pi\rangle
$$

with $\hat{\boldsymbol{\epsilon}}$, and using the commutation relations, we find

$$
\hat{\boldsymbol{\epsilon}} \hat{H}|E, \pi\rangle=\hat{H} \hat{\boldsymbol{\epsilon}}|E, \pi\rangle=E \hat{\boldsymbol{\epsilon}}|E, \pi\rangle .
$$

Hence both $|E, \pi\rangle$ and $\hat{\boldsymbol{\epsilon}}|E, \pi\rangle$ are eigenfunctions of $\hat{H}$ with the same eigenvalue $E$. The hydrogenic atom has thus a parity degeneracy, since $\hat{P}|E, \pi\rangle=\pi|E, \pi\rangle$, and $\hat{P} \hat{\epsilon}|E, \pi\rangle=$ $-\pi \hat{\boldsymbol{\epsilon}}|E, \pi\rangle, \hat{\boldsymbol{\epsilon}}$ being a polar vector. This establishes the connection between the constancy of $\langle\hat{\boldsymbol{\epsilon}}\rangle$ and degeneracies in hydrogenic atoms.

Two significant consequences of this degeneracy are as follows.

(a) The appearance of hybrid electron orbitals, i.e., linear combinations of states of the same energy but opposite parity, which has a bearing on the chemical properties of atoms.

(b) A linear Stark effect, rather than a quadratic one, ${ }^{4}$ in all states of a hydrogen atom in an external electric field, except the ground state, $\Psi_{n=1, l=0}$, which is the only nondegenerate one.

It should be noted in passing that by virtue of the fact that $\hat{\boldsymbol{\epsilon}}$ anticommutes with $\hat{P}$, and by Corollary 3 in Sec. II, we obtain the result $\hat{\epsilon} \Psi_{1,0}=0$, i.e., the Runge-Lenz operator annihilates the ground state. This can be easily verified by a direct calculation.

Finally, relativistic-order effects, such as a spin-orbit force, would upset the conditions in the main theorem in Sec. II, and could remove the degeneracies, at least partially, and invalidate the constancy of the direction of the major axis of the orbit. As is well known however, these relativistic effects are small and, hence, the deviations from the results established earlier are not significant. 


\section{B. Landau levels for a charged particle in a constant magnetic field $B$}

We consider a particle of mass $m$ and charge $q$ in a constant and uniform magnetic field in the $z$ direction, $\mathbf{B}=B \hat{e}_{z}$. Classically, the Hamiltonian for the system is

$$
H=\Pi^{2} / 2 m, \quad \Pi=\hat{\mathbf{p}}-(q / c) \mathbf{A},
$$

where $\mathbf{A}$ is the vector potential, i.e., $\mathbf{B}=\boldsymbol{\nabla} \times \mathbf{A}$, and $p$ and $\Pi$ are the canonical and kinetic momenta, respectively. The particle experiences no force along the $z$ axis.

The quantal Hamiltonian on the $x-y$ plane, $\hat{H}_{\perp}=\left(\Pi_{x}^{2}+\Pi_{y}^{2}\right) / 2 m$, is known to describe quantized motion, i.e., it has discrete eigenvalues $E_{n}$,

$$
E_{n}=\hbar \omega_{c}\left(n+\frac{1}{2}\right), \quad n=0,1,2, \ldots
$$

with $\omega_{c}=q B / m c$, the cyclotron frequency. These are the celebrated Landau levels whose derivation is described in standard texts, ${ }^{5}$ and will not be reviewed here. Our concern is to investigate the degeneracies in this spectrum, and their relation to constants of the motion, on the basis of the general theorem and corollaries described in Sec. II.

Returning momentarily to the classical regime, and using the fact that the magnetic field $B$ is constant, we may write the Lorenz force

$$
d \Pi / d t=(q / c) \mathbf{v} \times \mathbf{B}
$$

in the form

$$
d / d t[\mathbf{\Pi}-(q / c) \mathbf{r} \times \mathbf{B}]=0
$$

which shows that

$$
[\Pi-(q / c) \mathbf{r} \times \mathbf{B}]=\mathrm{a} \text { constant of the motion. }
$$

With $B$ along the $z$ axis,

$$
\begin{aligned}
& (q B / c) y-\Pi_{x}=(q B / c)\left[y-(c / q B) \Pi_{x}\right]=\text { constant, } \\
& (q B / c) x+\Pi_{y}=(q B / c)\left[x+(c / q B) \Pi_{y}\right]=\text { constant. }
\end{aligned}
$$

We use the above constants to define the components of a constant vector $\mathbf{R}$ perpendicular to $\mathbf{B}$, by

$$
R_{x}=x+(c / q B) \Pi_{y} \quad R_{y}=y-(c / q B) \Pi_{x}
$$

so that

$$
\begin{aligned}
\left(x-R_{x}\right)^{2}+\left(y-R_{y}\right)^{2} & =(c / q B)^{2}\left(\Pi_{x}^{2}+\Pi_{y}^{2}\right) \\
& =(c / q B)^{2} \Pi^{2} \\
& =\left(2 m c^{2} / q^{2} B^{2}\right) H .
\end{aligned}
$$

Hence the vector $\mathbf{R}$, a constant of the motion on the $x-y$ plane, defines the center of a circle on this plane.

Now, making the transition to quantum theory and defining operators $\hat{R}_{x}$ and $\hat{R}_{y}$ in terms of operators $\hat{x}, \hat{y}, \hat{\Pi}_{x}, \hat{\Pi}_{y}$, it is straightforward to show that

$$
\left[\hat{\Pi}_{x}, \hat{\Pi}_{y}\right]=i \hbar(q / c) B
$$

and

$$
\begin{aligned}
& {\left[\hat{H}, \hat{R}_{x}\right]=0, \quad\left[\hat{H}, \hat{R}_{y}\right]=0,} \\
& {\left[\hat{R}_{x}, \hat{R}_{y}\right]=-i \hbar c / q B=\text { constant number. }}
\end{aligned}
$$

Hence, not only are the requirements of Eqs. (3) and (4) satisfied by $\hat{H}, \hat{R}_{x}$, and $\hat{R}_{y}$, but also the commutator of $\hat{R}_{x}$ and $\hat{R}_{y}$ corresponding to two constants of the motion is a constant number. The consequence of this is that the entire spectrum of eigenvalues of the Hamiltonian is degenerate, and the degree of degeneracy for each eigenvalue is infinite. This is the explanation for the infinite degeneracy of the Landau levels which, as is well known, has important consequences for the behavior of conduction electrons in the presence of an external magnetic field.

Finally, we may note the classical analog of these degeneracies. As noted earlier, the values of the constants $R_{x}$ and $R_{y}$ define the position of the center of the classical circle described by the charged particle, but the energy of the particle is independent of the specific location of this center. ${ }^{6}$

\section{CONCLUSION}

By invoking a general theorem, and corollaries, regarding the commutator algebra of the Hamiltonian operator of quantal systems and operators corresponding to their constants of motion, as discussed in Sec. II, we are able to anticipate important properties of the systems without detailed solutions of their equations of motion. We have demonstrated the effectiveness of this method in the case of two important systems, i.e., hydrogenlike atoms and the Landau levels of charged particles in constant magnetic fields.

\section{ACKNOWLEDGMENTS}

This work was partially supported by the Department of Energy (S.F.) and by NSF Grant No. PHY-9213738 (E.H.).

\footnotetext{
${ }^{1}$ E. Merzbacher, Quantum Mechanics (Wiley, New York, 1961), p. 341. ${ }^{2}$ R. Shankar, Principles of Quantum Mechanics (Plenum, New York, 1980), pp. 369-370; G. Baym, Lectures on Quantum Mechanics (Benjamin, New York, 1969), p. 175; L. I. Schiff, Quantum Mechanics, 3rd ed. (McGrawHill, New York, 1968) pp. 235-237.

${ }^{3}$ H. Goldstein, Classical Mechanics, 2nd ed. (Addison-Wesley, Reading, MA, 1980), Chap. 3.

${ }^{4}$ R. Shankar, Principles of Quantum Mechanics (Plenum, New York, 1980), pp. $468,474$.

${ }^{5} \mathrm{C}$. Cohen-Tannoutji, B. Diu, and F. Laloe, Quantum Mechanics (Wiley, New York, 1977), p. 742.

${ }^{6}$ For a discussion of degeneracies in classical systems, see $\mathrm{S}$. Weigert and H. Thomas, "Classical degeneracy and the existence of additional constants of motion," Am. J. Phys. 61, 272-277 (1993).
}

\section{ASKING AGAIN AND AGAIN}

Whether or not the final laws of nature are discovered in our lifetime, it is a great thing for us to carry on the tradition of holding nature up to examination, of asking again and again why it is the way it is.

Steven Weinberg, Dreams of a Final Theory (Pantheon Books, New York, 1992), p. 275. 